\title{
The Transient and Asymptotic Moments for the Random Mission Time of a System
}

\author{
Los momentos transitorios y estables para el tiempo de misión de un sistema
}

\author{
Álvaro Calvache ${ }^{\mathrm{a} *}$ \\ Viswanathan Arunachalam ${ }^{\mathrm{b}}$ \\ Selvamuthu Dharmaraja ${ }^{c}$
}

Recepción: 27-ene-2016

Aceptación: 02-jun-2016

\begin{abstract}
In this paper, we study fault tolerant systems having one or more components and its system availability over the random mission time. The mission time is the time that elapses since the initial operation of the system until its cumulative working time achieves a predetermined fixed time. The main objective of this paper is to obtain the transient and asymptotic moments for the random mission time of the system availability subject to failures, as well as its distribution function, by using the theory of travel time distributions of a mobile, which passes through a finite number of paths wherein the average speed of the mobile varies from a path to another. A numerical example is presented to show the usefulness of the proposed model.
\end{abstract}

Key words: Mission time, Cumulative up-time, Transients moments, Asymptotic moments.

\section{Resumen}

En este artículo se estudian sistemas tolerantes a fallas con uno o más componentes, y su disponibilidad durante el tiempo aleatorio de misión. El tiempo de misión es aquél que transcurre desde la operación inicial del sistema hasta que su tiempo acumulado de trabajo alcanza un tiempo fijo predeterminado. El objetivo principal del artículo es la obtención de los momentos transitorios y estables del tiempo de misión de la disponibilidad del sistema sujeto a fallas, así como el análisis de su función de distribución, mediante el uso de la teoría de las distribuciones de tiempo de viaje de un móvil, que transita por un número finito de caminos, en los que la velocidad promedio del móvil varía de camino a camino. Un ejemplo numérico se presenta para mostrar la utilidad del modelo propuesto.

Palabras clave: Tiempo de misión, Tiempo acumulado en operación, Momentos transitorios, Momentos estables.

\footnotetext{
${ }^{a}$ Universidad Pedagógica y Tecnológica de Colombia.

*e-mail:alvaro.calvache@uptc.edu.co

${ }^{b}$ Universidad Nacional de Colombia.

${ }^{\mathrm{c}}$ Indian Institute of Technology Delhi, New Delhi, India.
} 


\section{Introduction}

This paper studies systems having one or more components, which can be arranged in parallel or series. The characteristic of our systems is that when system fails, they can be repaired, and while the systems are repaired, the they stop working. These systems can be classified into those that are fault tolerant and those that are not fault tolerant. Fault tolerant systems are those that do not compromise the entire enterprise. For example, systems for online transaction processing or systems for control process. These require an almost continuous operation and they tolerate short downtime. The preventive and corrective maintenance is generally allowed in these systems. On the other hand, systems that are not fault tolerant are those that provide a high reliability over a long period of the mission time, like those used in aerospace and in aviation. The system cannot be tolerated during downtime and the system reliability is an appropriate measure, since it is impossible to achieve desired purpose if the system fails before completion. In such cases, it is important and is more useful to analyze the transient or time dependent behavior of the system as failures occur occasionally, as compared to the steady state behavior. For fault tolerant systems, it is more appropriate to consider the time for which the system is in operation. Therefore, in these systems the most appropriate measure of evaluation is that the fraction of time the system is operating, which is also called availability of the system. Goyal and Tantawi [9] presented a complete analysis about a success (risk) measure of guaranteed availability.

De Souza e Silva and Gail [7] analyzed repairable fault tolerant systems. The system is modeled as a homogeneous Markov process. The distribution of cumulative up-time, that is, the distribution of the total time during which the system was in operation over a finite interval time is evaluated numerically. In this evaluation, the capability to specify error tolerances in advance, numerical stability and simplicity of implementation are applied. Sericola [15] extended the work of [7] and obtained a closedform solution for the distribution of the total time spent in a subset of states of a homogeneous Markov process during a finite time period. The results were applied to a fault tolerant system. Rubino and Sericola [14] considered a repairable computer system with alternating time periods during which it delivers uptime process and downtime process. The cumulative distribution of the uptime for the $n$th period is obtained. Donatiello and Iyer [8] presented a solution using Laplace transform of the distribution of availability using a semi-Markov process. Recently Arunachalam et al. [1] studied some useful approximation methods for the availability function.

Berry and Belmont [3] summarized different methods for analyzing the distributions of vehicle speeds and travel times. They found the relationship between speeds and travel times, set forth applications to preliminary data and suggested which techniques of analysis were best suited to the requirements of the engineers. Arunachalam and Dharmaraja [2], and Kharoufeh and Gautam [10] described a fluid queueing model as one of the stochastic systems which determines the amount of fluid contained in the buffer at any time instant. The system was modeled as a continuous fluid that enters a buffer and then leaves the buffer through a channel with a constant output capacity. Also, exists an external process, called the random environment process that modulates the input of fluid to the buffer and the states of this process dictates the rate at which fluid flows into the buffer. Kharoufeh and Gautam presented a model that described the time of travel of a vehicle for a link of fixed length. The system was controlled by a random environment process that determined the speed of the vehicle in each one of the states. Then, they realized that the two models could be attempted with the same methodology, and finally some results in the first model are used to obtain new results for the second model. Note that, these results presented by them have been complemented with others, such as those shown by Vanajakshi et al. [16], who gave an important application introducing at real-time short-term prediction of travel time for intelligent transportation systems. It should be noted that D'Angelo et al. [6] applied time series techniques for short-term travel time prediction. Also, Chen and Chien [5] analyzed the factors that would have an impact on the prediction accuracy about short-term travel time. On this point, it is important to note that Roden [13] identified potential alternative methods of addressing the travel time issue and he estimated the pros and cons of each method.

In this paper, we focus the availability analysis 
of the systems in which the random time required to accomplish the mission. The main idea is to consider for the distribution of system availability, that the cumulative time in system uptime is similar to the distance accumulated by a vehicle in a period of time. Then the result of the Laplace-Stieltjes transform for the distribution of the availability obtained by Donatiello and Iyer [8] is presented, but by using the methodology utilized by Kharoufeh and Gautam [10]. The contribution of the paper lies in the new results such as transient and asymptotic moments of the mission time.

The paper is organized as follows. In Section 2 , the description of the availability model for the system is presented. The solution in Laplace and Laplace-Stieltjes transforms for the differential equation of the underlying model is derived. Also, the results for the transients and asymptotic moments are presented. In Section 3, the alternative approaches are presented by using the probabilistic reasoning of the moments and the moment generating function of the mission time. The results obtained in Section 2 are graphically illustrated in Section 4.

\section{Distribution of System Availability}

In this section, we study the faulty tolerant systems having one or more components. We are interested to study the ratio of time that the system is working between the total time since the system started. This proportion is a random variable and it is known by the system availability. We now study the behavior of the distribution function of the system availability.

\subsection{Description of the Model}

The operating and the failure state of the system is modeled as a continuous time Markov chain (CTMC) with two states, namely, down state and up state. The aim is to determine the behavior of the random time of the system is working for an amount fixed of time. We shall use the following notations:

1. $\left\{Z_{t}, t \geq 0\right\}$ : CTMC which describes if the system is in up state or in down state at time $t$.

2. $S=\{0,1\}$ : State space of $\left\{Z_{t}, t \geq 0\right\}$. 0 represents the down state of the system and 1 is indicates of the up/operative state of the system.

3. $C_{t}$ : If we call $\{Z=1\}=\left\{t \geq 0 \mid Z_{t}=1\right\}$, then the cumulative up-time is defined by

$$
C_{t}=\int_{0}^{t} I_{\{Z=1\}}(v) d v,
$$

where $I_{A}$ represents the indicator function over the event $A$. Also, $C=\left\{C_{t}, t \geq 0\right\}$ is the cumulative up-time process.

4. $\mathbf{Q}=\left[\begin{array}{cc}-\lambda_{0} & \lambda_{0} \\ \lambda_{1} & -\lambda_{1}\end{array}\right]$ is the infinitesimal generator for the process $\left\{Z_{t}, t \geq 0\right\}$.

5. $V_{Z_{t}}=\frac{d}{d t} C_{t}$ is the rate at which accumulates the time when the system is up, in a time $t \geq 0$, therefore $V_{1}=1$ and $V_{0}=0$.

6. $\mathbf{V}=\left[\begin{array}{ll}0 & 0 \\ 0 & 1\end{array}\right]=\operatorname{diag}\left(V_{0}, V_{1}\right)$ is called the speed diagonal matrix[11].

7. $\mathbf{z}_{\mathbf{0}}$ : The initial distribution, that is,

$$
\mathbf{z}_{\mathbf{0}}=\left(P_{r}\left\{Z_{0}=0\right\}, P_{r}\left\{Z_{0}=1\right\}\right)=(0,1),
$$

since, we have supposed that the system starts in up-state.

8. $H_{i}(x, t)$ for $i \in S$ : Probability that at time $t$, the system is in state $i$, and that in the time interval $[0, t)$, the time spent by the system in up state is less than the time $x$. Hence,

$$
H_{i}(x, t)=P_{r}\left\{C_{t} \leq x, Z_{t}=i\right\}, \quad i=0,1 .
$$

9. $\mathbf{H}(x, t)$ : Row vector

$$
\mathbf{H}(x, t)=\left[H_{0}(x, t), H_{1}(x, t)\right] .
$$

10. $M_{x}$ : Given a fixed time $x \geq 0$, the mission time for $x$ is

$$
M_{x}=\inf \left\{t \geq 0 \mid C_{t} \geq x\right\} .
$$

$M_{x}$ is the random time that has elapsed since the initial operation of the system, for that its cumulative time working is $x$. This time $x$ is also called the length of the mission.

11. $G(x, t)=F_{M_{x}}(t)$ : For $x$ fixed, it is the cumulative distribution function for $M_{x}$, i.e.,

$$
G(x, t)=F_{M_{x}}(t)=P\left\{M_{x} \leq t\right\} .
$$

12. $R(x, t)=F_{C_{t}}(x)$ : For a fixed $t$, it is the cumulative distribution function for $C_{t}$, i.e.,

$$
R(x, t)=F_{C_{t}}(x)=P_{r}\left\{C_{t} \leq x\right\} .
$$


13. $m_{r}(x)$ : The $r^{\text {th }}$ moment of $M_{x}$, that is,

$$
m_{r}(x)=E\left[M_{x}^{r}\right], \quad \text { for } x \geq 0,
$$

if this value exists.

14. I and 1: The identity matrix and the column vector of the only ones, respectively.

\subsection{The Availability Model}

The following three Lemmas are required to evaluate the distribution of the mission time.

Theorem 1. $\left\{M_{x}<t\right\}=\left\{C_{t}>x\right\}$ for $t, x \geq 0$.

Proof. Follows from the definition of $M_{x}$.

Theorem 2. $G(x, t)=1-R(x, t)=1-\sum_{i \in S} H_{i}(x, t)$.

Proof. $G(x, t)=P_{r}\left\{M_{x} \leq t\right\}=P_{r}\left\{C_{t} \geq x\right\}$, by Lemma 1, and then $G(x, t)=1-P_{r}\left\{C_{t} \leq x\right\}=$ $1-\sum_{i \in S} P_{r}\left\{C_{t} \leq x, Z_{t}=i\right\}=1-\sum_{i \in S} H_{i}(x, t)$.

Theorem 3. The joint probability distribution $\mathbf{H}(x, t)$ satisfies the system of the partial differential equations:

$$
\begin{aligned}
\frac{\partial H_{0}(x, t)}{\partial t} & =-\lambda_{0} H_{0}(x, t)+\lambda_{1} H_{1}(x, t) \\
\frac{\partial H_{1}(x, t)}{\partial t}+\frac{\partial H_{1}(x, t)}{\partial x} & =\lambda_{0} H_{0}(x, t)-\lambda_{1} H_{1}(x, t)
\end{aligned}
$$

with initial conditions:

$$
H_{0}(x, 0)=0 \text { and } H_{1}(x, 0)=1 .
$$

Proof. Since that $C_{t}$ is a differentiable function in almost everywhere, therefore:

$$
C_{t+\delta}=C_{t}+\delta \frac{d}{d t} C_{t}+o(\delta)=C_{t}+\delta V_{Z_{t}}+o(\delta)
$$

where $\lim _{\delta \rightarrow 0} o(\delta) / \delta=0$. For $j \in S$,

$$
\begin{aligned}
P_{r}\left\{C_{t+\delta} \leq x, Z_{t}=j\right\} & =P_{r}\left\{C_{t}+\delta V_{Z_{t}}+o(\delta) \leq x, Z_{t}=j\right\} \\
& =P_{r}\left\{C_{t} \leq x-\delta V_{Z_{t}}+o(\delta), Z_{t}=j\right\} \\
& =P_{r}\left\{C_{t} \leq x-\delta V_{j}+o(\delta), Z_{t}=j\right\} \\
& =H_{j}\left(x-\delta V_{j}, t\right)+o(\delta) .
\end{aligned}
$$

Also,

for all $t \geq 0, C_{t}$ is independent with $Z_{t}$, in fact,

$$
\begin{aligned}
P_{r}\left\{C_{t}\right. & \left.\leq x \mid Z_{t}=a\right\} \\
& =P_{r}\left\{\int_{0}^{t} I_{\{Z=1\}}(v) d v \leq x \mid Z_{t}=a\right\} \\
& =P_{r}\left\{\int_{0}^{t} I_{\{Z=1\}}(v) d v \leq x\right\}=P_{r}\left\{C_{t} \leq x\right\} .
\end{aligned}
$$

Thus,

$$
\begin{aligned}
H_{0}(x, t+\delta)= & P_{r}\left\{C_{t+\delta} \leq x, Z_{t+\delta}=0\right\} \\
= & \sum_{j \in S} P_{r}\left\{C_{t+\delta} \leq x, Z_{t+\delta}=0, Z_{t}=j\right\} \\
= & \sum_{j \in S} P_{r}\left\{Z_{t+\delta}=0 \mid C_{t+\delta} \leq x, Z_{t}=j\right\} \\
& \times P_{r}\left\{C_{t+\delta} \leq x, Z_{t}=j\right\} \\
(\stackrel{6)}{=} & \sum_{j \in S} P_{r}\left\{Z_{t+\delta}=0 \mid Z_{t}=j\right\} \\
& \times P_{r}\left\{C_{t+\delta} \leq x, Z_{t}=j\right\}
\end{aligned}
$$

and by (5),

$$
\begin{aligned}
= & \sum_{j \in S} P_{r}\left\{Z_{t+\delta}=0 \mid Z_{t}=j\right\} \\
& \times\left(H_{j}\left(x-\delta V_{j}, t\right)+o(\delta)\right) \\
= & \left(1-\lambda_{0} \delta+o(\delta)\right) \\
& \times\left(H_{0}\left(x-\delta V_{0}, t\right)+o(\delta)\right) \\
& +\left(\lambda_{1} \delta+o(\delta)\right) \\
& \times\left(H_{1}\left(x-\delta V_{1}, t\right)+o(\delta)\right) \\
= & \left(1-\lambda_{0} \delta\right) \times H_{0}(x, t)+\lambda_{1} \delta \\
& \times H_{1}(x-\delta, t)+o(\delta) \\
= & H_{0}(x, t)-\lambda_{0} \delta \cdot H_{0}(x, t)+\lambda_{1} \delta \\
& \times H_{1}(x-\delta, t)+o(\delta) .
\end{aligned}
$$

Then,

$$
\frac{\partial}{\partial t} H_{0}(x, t)=\lim _{\delta \rightarrow 0} \frac{H_{0}(x, t+\delta)-H_{0}(x, t)}{\delta}
$$

and by (7),

$$
\begin{aligned}
& =\lim _{\delta \rightarrow 0}\left[-\lambda_{0} H_{0}(x, t)+\lambda_{1} H_{1}(x-\delta, t)+\frac{o(\delta)}{\delta}\right] \\
& =-\lambda_{0} H_{0}(x, t)+\lambda_{1} H_{1}(x, t) .
\end{aligned}
$$


Hence, equation (1) is satisfied. Now,

$$
\begin{aligned}
H_{1}(x, t+\delta)= & P_{r}\left\{C_{t+\delta} \leq x, Z_{t+\delta}=1\right\} \\
= & \sum_{j \in S} P_{r}\left\{C_{t+\delta} \leq x, Z_{t+\delta}=1, Z_{t}=j\right\} \\
= & \sum_{j \in S} P_{r}\left\{Z_{t+\delta}=1 \mid C_{t+\delta} \leq x, Z_{t}=j\right\} \\
& \times P_{r}\left\{C_{t+\delta} \leq x, Z_{t}=j\right\} \\
(\stackrel{6)}{=} & \sum_{j \in S} P_{r}\left\{Z_{t+\delta}=1 \mid Z_{t}=j\right\} \\
& \times P_{r}\left\{C_{t+\delta} \leq x, Z_{t}=j\right\}
\end{aligned}
$$

and by (5),

$$
\begin{aligned}
= & \sum_{j \in S} P_{r}\left\{Z_{t+\delta}=1 \mid Z_{t}=j\right\} \\
& \times\left(H_{j}\left(x-\delta V_{j}, t\right)+o(\delta)\right)
\end{aligned}
$$

(and since $\mathbf{Q}=\left[\begin{array}{cc}-\lambda_{0} & \lambda_{0} \\ \lambda_{1} & -\lambda_{1}\end{array}\right]$ is the infinitesimal generator for the process $\left\{Z_{t}, t \geq 0\right\}$ ),

$$
\begin{aligned}
= & \left(\lambda_{0} \delta+o(\delta)\right) \cdot\left(H_{0}\left(x-\delta V_{0}, t\right)+o(\delta)\right) \\
& +\left(1-\lambda_{1} \delta+o(\delta)\right) \cdot\left(H_{1}\left(x-\delta V_{1}, t\right)+o(\delta)\right)
\end{aligned}
$$

and as $V_{0}=0, V_{1}=1$,

$$
\begin{gathered}
=\left(\lambda_{0} \delta\right) \cdot H_{0}(x, t)+\left(1-\lambda_{1} \delta\right) \cdot H_{1}(x-\delta, t)+o(\delta) \\
=\lambda_{0} \delta \cdot H_{0}(x, t)+H_{1}(x-\delta, t) \\
\quad-\lambda_{1} \delta \cdot H_{1}(x-\delta, t)+o(\delta) .
\end{gathered}
$$

Then,

$$
\begin{aligned}
\frac{\partial}{\partial t} H_{1}(x, t)= & \lim _{\delta \rightarrow 0} \frac{H_{1}(x, t+\delta)-H_{1}(x, t)}{\delta} \\
\stackrel{(8)}{=} & \lim _{\delta \rightarrow 0}\left[\lambda_{0} H_{0}(x, t)-\lambda_{1} H_{1}(x-\delta, t)\right. \\
& \left.+\frac{H_{1}(x-\delta, t)-H_{1}(x, t)}{\delta}+\frac{o(\delta)}{\delta}\right] \\
= & \lambda_{0} H_{0}(x, t)-\lambda_{1} H_{1}(x, t)-\frac{\partial}{\partial x} H_{1}(x, t) .
\end{aligned}
$$

Hence (2) holds. Besides, since the process is assumed to starts in the up state, therefore:

$$
\begin{aligned}
& H_{0}(x, 0)=P_{r}\left\{C_{0} \leq x, Z_{0}=0\right\} \\
& \quad \stackrel{(6)}{=} \operatorname{Pr}\left\{C_{0} \leq x\right\} \cdot P_{r}\left\{Z_{0}=0\right\}=1 \cdot 0=0,
\end{aligned}
$$

and

$$
\begin{aligned}
& H_{1}(x, 0)=P_{r}\left\{C_{0} \leq x, Z_{0}=1\right\} \\
& \quad \stackrel{(6)}{=} P_{r}\left\{C_{0} \leq x\right\} \cdot P_{r}\left\{Z_{0}=1\right\}=1 \cdot 1=1 .
\end{aligned}
$$

Hence we obtain (3).
Remark 4. The equations (1) and (2), with initial conditions (3) can be written in the matrix form:

$$
\frac{\partial \mathbf{H}(x, t)}{\partial t}+\frac{\partial \mathbf{H}(x, t)}{\partial x} \mathbf{V}=\mathbf{H}(x, t) \mathbf{Q}
$$

with initial condition:

$$
\mathbf{H}(x, 0)=\mathbf{z}_{\mathbf{0}} .
$$

\subsection{The Laplace Transform and the Laplace-} Stieltjes Transform of the Distribution Function for $C_{t}$

We shall use the following notations in the results to follow:

1. If $f(t)$ is a real function, we denote by $f^{*}\left(s_{2}\right)$ its Laplace transform (LT), with respect to $t$ (if it exists), i.e.,

$$
f^{*}\left(s_{2}\right)=\int_{0}^{\infty} e^{-t s_{2}} f(t) d t
$$

2. And if $g(x)$ is a real function, we denote by $\widetilde{g}\left(s_{1}\right)$ its Laplace-Stieltjes transform (LST), with respect to $x$ (if it exists), i.e.,

$$
\widetilde{g}\left(s_{1}\right)=\int_{0}^{\infty} e^{-x s_{1}} d g(x) .
$$

3. $\widetilde{\mathbf{H}}^{*}\left(s_{1}, s_{2}\right)$ represents the two-dimensional vector, double Laplace and Laplace-Stieltjes transform (LT-LST) of $\mathbf{H}(x, t)$ (if it exists), i.e.,

$$
\widetilde{\mathbf{H}}^{*}\left(s_{1}, s_{2}\right):=\int_{0}^{\infty} e^{-x s_{1}} d \mathbf{H}^{*}\left(x, s_{2}\right) .
$$

4. If $\mathbf{H}$ is differentiable, then

$$
\widetilde{\mathbf{H}}^{*}\left(s_{1}, s_{2}\right)=\int_{0}^{\infty} \int_{0}^{\infty} e^{-x s_{1}-t s_{2}} \frac{\partial}{\partial x} \mathbf{H}(x, t) d t d x .
$$

Theorem 5. In an interval of time $[0, t)$, the double Laplace and Laplace-Stieltjes transform of the distribution function for $C_{t}$, that is, the LT-LST of $R(x, t)=P_{r}\left\{C_{t} \leq x\right\}$ is given by:

$$
\widetilde{R}^{*}\left(s_{1}, s_{2}\right)=\frac{s_{2}+\lambda_{0}+\lambda_{1}}{\left(s_{2}+\lambda_{0}\right)\left(s_{1}+s_{2}+\lambda_{1}\right)-\lambda_{0} \lambda_{1}} .
$$


Proof. Taking LT, with respect to $t$ in (1) and for using the first part of the initial conditions in (3), we obtain:

$$
s_{2} H_{0}^{*}\left(x, s_{2}\right)=-\lambda_{0} H_{0}^{*}\left(x, s_{2}\right)+\lambda_{1} H_{1}^{*}\left(x, s_{2}\right)
$$

and we have:

$$
H_{1}^{*}\left(x, s_{2}\right)=\frac{\lambda_{0}+s_{2}}{\lambda_{1}} H_{0}^{*}\left(x, s_{2}\right) .
$$

Now, taking LT with respect to $t$ in (2) and for using the second part of the initial conditions in (3), we obtain:

$$
\begin{aligned}
& s_{1} H_{1}^{*}\left(x, s_{2}\right)-1+\frac{\partial}{\partial x} H_{1}^{*}\left(x . s_{2}\right)= \\
& \lambda_{0} H_{0}^{*}\left(x, s_{2}\right)-\lambda_{1} H_{1}^{*}\left(x, s_{2}\right)
\end{aligned}
$$

and replacing (14) in (15):

$$
\begin{gathered}
\frac{s_{2}\left(\lambda_{0}+s_{2}\right)}{\lambda_{1}} H_{0}^{*}\left(x, s_{2}\right)-1+\frac{\lambda_{0}+s_{2}}{\lambda_{1}} \frac{\partial}{\partial x} H_{0}^{*}\left(x, s_{2}\right)= \\
\lambda_{0} H_{0}^{*}\left(x, s_{2}\right)-\left(\lambda_{0}+s_{2}\right) H_{0}^{*}\left(x, s_{2}\right) .
\end{gathered}
$$

Taking LT with respect to $x$ with $H_{1}^{*}\left(0, s_{2}\right)=0$, we obtain:

$$
\begin{aligned}
& \frac{\left(s_{1}+s_{2}\right)\left(\lambda_{0}+s_{2}\right)}{\lambda_{1}} H_{0}^{* *}\left(s_{1}, s_{2}\right)-\frac{1}{s_{1}}= \\
& \quad \lambda_{0} H_{0}^{* *}\left(s_{1}, s_{2}\right)-\left(\lambda_{0}+s_{2}\right) H_{0}^{* *}\left(s_{1}, s_{2}\right)
\end{aligned}
$$

and solving for $H_{0}^{* *}\left(s_{1}, s_{2}\right)$ :

$$
H_{0}^{* *}\left(s_{1}, s_{2}\right)=\frac{\lambda_{1}}{s_{1}\left[\left(s_{2}+\lambda_{0}\right)\left(s_{1}+s_{2}+\lambda_{1}\right)-\lambda_{0} \lambda_{1}\right]} .
$$

Taking LT with respect to $x$ in (14) and by (16):

$$
H_{1}^{* *}\left(s_{1}, s_{2}\right)=\frac{\lambda_{0}+s_{2}}{s_{1}\left[\left(s_{2}+\lambda_{0}\right)\left(s_{1}+s_{2}+\lambda_{1}\right)-\lambda_{0} \lambda_{1}\right]} .
$$

Hence, we get

$$
\begin{aligned}
& \widetilde{H}_{0}^{*}\left(s_{1}, s_{2}\right)=\frac{\lambda_{1}}{\left(s_{2}+\lambda_{0}\right)\left(s_{1}+s_{2}+\lambda_{1}\right)-\lambda_{0} \lambda_{1}} \\
& \widetilde{H}_{1}^{*}\left(s_{1}, s_{2}\right)=\frac{\lambda_{0}+s_{2}}{\left(s_{2}+\lambda_{0}\right)\left(s_{1}+s_{2}+\lambda_{1}\right)-\lambda_{0} \lambda_{1}} .
\end{aligned}
$$

Remember that, $R(x, t)=P_{r}\left\{C_{t} \leq x\right\}$ (Probability that in interval the time $[0, t)$, the amount of time spent by the system in up-state is less or equal to time $x$.) Thus,

$$
\begin{aligned}
R(x, t) & =P_{r}\left\{C_{t} \leq x, Z_{t}=0\right\}+P_{r}\left\{C_{t} \leq x, Z_{t}=1\right\} \\
& =H_{0}(x, t)+H_{1}(x, t) .
\end{aligned}
$$

Then,

$$
\widetilde{R}^{*}\left(s_{1}, s_{2}\right)=\widetilde{H}_{0}^{*}\left(s_{1}, s_{2}\right)+\widetilde{H}_{1}^{*}\left(s_{1}, s_{2}\right)
$$

and by (18), (19) and (20), we obtain the result in (13).

Remark 6. Note that the equations (18) and (19) can be written in matrix form as follows:

$$
\widetilde{\mathbf{H}}^{*}\left(s_{1}, s_{2}\right)=\mathbf{z}_{\mathbf{0}}\left[s_{2} \mathbf{I}+s_{1} \mathbf{V}-\mathbf{Q}\right]^{-1}
$$

In fact,

$$
\begin{gathered}
\mathbf{z}_{0}\left[s_{2} \mathbf{I}+s_{1} \mathbf{V}-\mathbf{Q}\right]^{-1} \\
=(0,1)\left(s_{2}\left[\begin{array}{ll}
1 & 0 \\
0 & 1
\end{array}\right]+s_{1}\left[\begin{array}{ll}
0 & 0 \\
0 & 1
\end{array}\right]-\left[\begin{array}{cc}
-\lambda_{0} & \lambda_{0} \\
\lambda_{1} & -\lambda_{1}
\end{array}\right]\right)^{-1} \\
=\frac{1}{\left(s_{2}+\lambda_{0}\right)\left(s_{1}+s_{2}+\lambda_{1}\right)-\lambda_{0} \lambda_{1}}\left[\lambda_{1}, s_{2}+\lambda_{0}\right]= \\
{\left[\widetilde{H}_{0}^{*}\left(s_{1}, s_{2}\right), \widetilde{H}_{1}^{*}\left(s_{1}, s_{2}\right)\right]=\widetilde{\mathbf{H}}^{*}\left(s_{1}, s_{2}\right) .}
\end{gathered}
$$

\subsection{Transient Moments for the Mission Time $M_{x}$}

In this subsection, we answer to the following questions: Given a length of the mission $x \geq 0$, what is the expected value of the mission time $M_{x}$ ? What is its variability? Before we discuss some results that provide answer to these questions, we present the following remark:

Remark 7. Note that (13) coincides with the result obtained by Donatiello and Iyer [8], however, the their approach was based on renewal theoretic argument. We now give the closed form expression for $R(x, t)$, in the case that $\left\{Z_{t}, t \geq 0\right\}$ to be a Markov process:

$$
\begin{aligned}
& R(x, t)= \\
& 1-e^{-\lambda_{1} x}\left\{1+\frac{e^{-\lambda_{0}(t-x)}\left(2 \lambda_{1} x I_{0}[\alpha]+\frac{4 x^{2} I_{1}[\alpha] \sqrt{\lambda_{0} \lambda_{1}}}{\sqrt{x(t-x)}}\right)-2 x \lambda_{1}}{2 \lambda_{1} x-1}\right\},
\end{aligned}
$$

where $\alpha=2 \sqrt{\lambda_{0} \lambda_{1} x(t-x)}$ and $I_{i}[\alpha]$ is the modified Bessel function of order $i$. 
Theorem 8. Given the fixed time $x \geq 0$, then

$$
\begin{aligned}
E\left[M_{x}\right] & =\frac{\lambda_{0}+\lambda_{1}}{\lambda_{0}} x, \\
\operatorname{Var}\left[M_{x}\right] & =2 \frac{\lambda_{1}}{\lambda_{0}^{2}} x .
\end{aligned}
$$

Proof. First, by Lemma 2,

$$
G(x, t)=1-\sum_{i \in S} H_{i}(x, t),
$$

and by properties of the Laplace Transform and the Laplace-Stieltjes transform, we obtain:

$$
\begin{gathered}
\widetilde{G}\left(x, s_{2}\right)=-G(x, 0)+s_{2} G^{*}\left(x, s_{2}\right) \\
=-\left[1-\sum_{i \in S} H_{i}(x, 0)\right]+s_{2}\left[\frac{1}{s_{2}}-\sum_{i \in S} H_{i}^{*}\left(x, s_{2}\right)\right] \\
=-\left[1-\sum_{i \in S} P_{r}\left\{Z_{0}=i\right\}\right]+s_{2}\left[\frac{1}{s_{2}}-\sum_{i \in S} H_{i}^{*}\left(x, s_{2}\right)\right] \\
=1-s_{2} \sum_{i \in S} H_{i}^{*}\left(x, s_{2}\right) .
\end{gathered}
$$

For $r \in\{1,2, \ldots\}$, differentiating $r$ times with respect to $s_{2}$, by induction it is possible to demonstrate that,

$$
\begin{aligned}
& \frac{\partial^{r} \widetilde{G}\left(x, s_{2}\right)}{\partial s_{2}^{r}}= \\
& -\left[s_{2} \sum_{i \in S} \frac{\partial^{r} H_{i}^{*}\left(x, s_{2}\right)}{\partial s_{2}^{r}}+r \sum_{i \in S} \frac{\partial^{r-1} H_{i}^{*}\left(x, s_{2}\right)}{\partial s_{2}^{r-1}}\right] .
\end{aligned}
$$

On the other hand, note that if $F$ is a differentiable real function and if $f(t)=F^{\prime}(t)$ then $f^{*}(s)=\widetilde{F}(s)$ and therefore,

$$
\begin{aligned}
& \frac{d^{n} \widetilde{F}(s)}{d s^{n}}=\frac{d^{n} f^{*}(s)}{d s^{n}} \\
& \quad=\left[(-t)^{n} f(t)\right]^{*}(s)=\int_{0}^{\infty}(-t)^{n} f(t) e^{-t s} d t
\end{aligned}
$$

and hence,

$$
\begin{aligned}
\frac{d^{n} \widetilde{F}(0)}{d s^{n}} & =\frac{d^{n} f^{*}(0)}{d s^{n}} \\
& =\left[(-t)^{n} f(t)\right]^{*}(0)=\int_{0}^{\infty}(-t)^{n} f(t) d t .
\end{aligned}
$$

Then,

$$
\begin{aligned}
m_{r}(x)=E\left[M_{x}^{r}\right]=\int_{0}^{\infty} t^{r} \frac{\partial G(x, t)}{\partial t} d t & \\
& =(-1)^{r} \frac{\partial^{r} \widetilde{G}(x, 0)}{\partial s_{2}^{r}}
\end{aligned}
$$

and by (25),

$$
\begin{aligned}
& m_{r}(x)=(-1)^{r+1} r \sum_{i \in S} \frac{\partial^{r-1} H_{i}^{*}(x, 0)}{\partial s_{2}^{r-1}} \\
& =(-1)^{r+1} r\left[\frac{\partial^{r-1} H_{0}^{*}(x, 0)}{\partial s_{2}^{r-1}}+\frac{\partial^{r-1} H_{1}^{*}(x, 0)}{\partial s_{2}^{r-1}}\right]
\end{aligned}
$$

and taking LST on both sides,

$$
\begin{aligned}
& \widetilde{m}_{r}\left(s_{1}\right)= \\
& (-1)^{r+1} r\left[\frac{\partial^{r-1} \widetilde{H}_{0}^{*}\left(s_{1}, 0\right)}{\partial s_{2}^{r-1}}+\frac{\partial^{r-1} \widetilde{H}_{1}^{*}\left(s_{1}, 0\right)}{\partial s_{2}^{r-1}}\right] .
\end{aligned}
$$

Hence, by (18), (19) and (26), we obtain:

$$
\begin{aligned}
& \widetilde{m}_{1}\left(s_{1}\right)=\widetilde{H}_{0}^{*}\left(s_{1}, 0\right)+\widetilde{H}_{1}^{*}\left(s_{1}, 0\right) \\
= & \frac{\lambda_{1}}{\left(\lambda_{0}\right)\left(s_{1}+\lambda_{1}\right)-\lambda_{0} \lambda_{1}}+\frac{\lambda_{0}}{\left(\lambda_{0}\right)\left(s_{1}+\lambda_{1}\right)-\lambda_{0} \lambda_{1}} \\
& =\frac{\lambda_{0}+\lambda_{1}}{\lambda_{0} s_{1}} .
\end{aligned}
$$

Therefore,

$$
m_{1}(x)=\frac{\lambda_{0}+\lambda_{1}}{\lambda_{0}} x=E\left[M_{x}\right] .
$$

Also by (18), (19) and (26):

$$
\begin{aligned}
\widetilde{m}_{2}\left(s_{1}\right)=-2 & {\left[\frac{\partial \widetilde{H}_{0}^{*}\left(s_{1}, 0\right)}{\partial s_{2}}+\frac{\partial \widetilde{H}_{1}^{*}\left(s_{1}, 0\right)}{\partial s_{2}}\right] } \\
& =2\left(\frac{\lambda_{1}}{\lambda_{0}^{2} s_{1}}+\left(\frac{\lambda_{0}+\lambda_{1}}{\lambda_{0}}\right)^{2} \frac{1}{s_{1}^{2}}\right) .
\end{aligned}
$$

Therefore,

$$
m_{2}(x)=2 \frac{\lambda_{1}}{\lambda_{0}^{2}} x+\left(\frac{\lambda_{0}+\lambda_{1}}{\lambda_{0}}\right)^{2} x^{2}=E\left[M_{x}^{2}\right] .
$$

Finally,

$$
\operatorname{Var}\left[M_{x}\right]=E\left[M_{x}^{2}\right]-E\left[M_{x}\right]^{2}=2 \frac{\lambda_{1}}{\lambda_{0}^{2}} x .
$$




\subsection{Asymptotic Moments for the Mission Time} $M_{x}$

We now obtain the asymptotic moments of first and second order, for the mission time $M_{x}$.

\section{Corollary 9.}

$$
\begin{aligned}
& \lim _{x \rightarrow \infty} \frac{E\left[M_{x}\right]}{x}=\frac{\lambda_{0}+\lambda_{1}}{\lambda_{0}}, \text { and } \\
& \lim _{x \rightarrow \infty} \frac{E\left[M_{x}^{2}\right]}{x^{2}}=\left(\frac{\lambda_{0}+\lambda_{1}}{\lambda_{0}}\right)^{2} .
\end{aligned}
$$

Proof. This result follows from Theorem 8, more precisely from equations (27) and (28).

Remark 10. Kharoufeh and Gautam [11] found that if the process $\left\{Z_{t}, t \geq 0\right\}$ has a stationary distribution $\mathbf{p}$, the speed diagonal matrix $V$ is defined positive and $m_{2}^{\prime}(x) \rightarrow \infty$ as $x \rightarrow \infty$, then,

$$
\begin{array}{r}
\lim _{x \rightarrow \infty} \frac{m_{1}(x)}{x}=\frac{1}{\mathbf{p} \cdot \mathbf{V} \cdot \mathbf{1}}, \quad \text { and } \\
\lim _{x \rightarrow \infty} \frac{m_{2}(x)}{x^{2}}=\frac{1}{(\mathbf{p} \cdot \mathbf{V} \cdot \mathbf{1})^{2}} .
\end{array}
$$

Now, by taking

$$
\lim _{V_{0} \rightarrow 0^{+}} \frac{1}{\mathbf{p} \cdot \mathbf{V} \cdot \mathbf{1}} \quad \text { and } \lim _{V_{0} \rightarrow 0^{+}} \frac{1}{(\mathbf{p} \cdot \mathbf{V} \cdot \mathbf{1})^{2}},
$$

the results in (30) also are valid in our model, since the expressions in its denominators are continuous and $\lim _{V_{0} \rightarrow 0^{+}}(\mathbf{p} \cdot \mathbf{V} \cdot \mathbf{1}) \neq 0$. Hence, we can demonstrate (29), without using Theorem 8. In fact, for $t \geq 0$, let us denote $P_{i j}(t)=P_{r}\left\{Z_{t}=j \mid Z_{0}=i\right\}$, for $i, j \in S$ and the transition probability matrix $\mathbf{P}(t)=\left[P_{i j}(t)\right]_{i, j \in S}$. It is well known that (Refer [4]):

$$
\begin{gathered}
\mathbf{P}(t)= \\
{\left[\begin{array}{ll}
\frac{\lambda_{1}}{\lambda_{0}+\lambda_{1}}+\frac{\lambda_{0}}{\lambda_{0}+\lambda_{1}} e^{-\left(\lambda_{0}+\lambda_{1}\right) t} & \frac{\lambda_{0}}{\lambda_{0}+\lambda_{1}}-\frac{\lambda_{0}}{\lambda_{0}+\lambda_{1}} e^{-\left(\lambda_{0}+\lambda_{1}\right) t} \\
\frac{\lambda_{1}}{\lambda_{0}+\lambda_{1}}-\frac{\lambda_{1}}{\lambda_{0}+\lambda_{1}} e^{-\left(\lambda_{0}+\lambda_{1}\right) t} & \frac{\lambda_{0}}{\lambda_{0}+\lambda_{1}}+\frac{\lambda_{1}}{\lambda_{0}+\lambda_{1}} e^{-\left(\lambda_{0}+\lambda_{1}\right) t}
\end{array}\right]}
\end{gathered}
$$

and therefore,

$$
\mathbf{p}=\left[\frac{\lambda_{1}}{\lambda_{0}+\lambda_{1}}, \frac{\lambda_{0}}{\lambda_{0}+\lambda_{1}}\right]
$$

is a stationary distribution row vector for $\left\{Z_{t}, t \geq 0\right\}$. But,

$\mathbf{p} \cdot \mathbf{V} \cdot \mathbf{1}=\left[\frac{\lambda_{1}}{\lambda_{0}+\lambda_{1}}, \frac{\lambda_{0}}{\lambda_{0}+\lambda_{1}}\right]\left[\begin{array}{ll}0 & 0 \\ 0 & 1\end{array}\right]\left[\begin{array}{l}1 \\ 1\end{array}\right]=\frac{\lambda_{0}}{\lambda_{0}+\lambda_{1}}$.
Then, by the first part of (30),

$$
\lim _{x \rightarrow \infty} \frac{E\left[M_{x}\right]}{x}=\frac{1}{\mathbf{p} \cdot \mathbf{V} \cdot \mathbf{1}}=\frac{\lambda_{0}+\lambda_{1}}{\lambda_{0}} .
$$

Similarly, using the second part of (30), we obtain,

$$
\lim _{x \rightarrow \infty} \frac{E\left[M_{x}^{2}\right]}{x^{2}}=\left(\frac{\lambda_{0}+\lambda_{1}}{\lambda_{0}}\right)^{2} .
$$

\section{Corollary 11.}

$$
\lim _{x \rightarrow \infty} \frac{\operatorname{Var}\left[M_{x}\right]}{x}=2 \frac{\lambda_{1}}{\lambda_{0}^{2}} .
$$

Proof. The result follows from Theorem 8.

\section{Alternative Approaches}

In this section, we present some alternative approaches to derive the moments for the mission time $E\left[M_{x}^{k}\right]$, for $x>0$ and $k \in \mathbb{Z}^{+}$(if they exist), without using Lemma 3 and inverting transforms. First, we present a direct derivation of the moments of the mission time.

Lemma 12. For $x>0, h>0$ and $k \in \mathbb{Z}^{+}$,

$$
E\left[M_{x+h}^{k}\right]=\sum_{j=0}^{k}\left(\begin{array}{c}
k \\
j
\end{array}\right) E\left[M_{x}^{j}\right] E\left[M_{h}^{k-j}\right],
$$

if $E\left[M_{t}^{j}\right]$ exists, for all $t \geq 0$, for all $j=0,1, \ldots, k$.

Proof. By the strong Markov property, we have

$$
E\left[M_{x+h}^{k} \mid M_{x}=z\right]=E\left[\left(z+M_{h}\right)^{k}\right],
$$

then,

$$
\begin{aligned}
E\left[M_{x+h}^{k}\right] & =E\left[E\left[M_{x+h}^{k} \mid M_{x}\right]\right] \\
& =\int_{0}^{\infty} E\left[M_{x+h}^{k} \mid M_{x}=z\right] d F_{M_{x}}(z) \\
& \stackrel{(33)}{=} \int_{0}^{\infty} E\left[\left(z+M_{h}\right)^{k}\right] d F_{M_{x}}(z), \\
& =\int_{0}^{\infty} E\left[\sum_{j=0}^{k}\left(\begin{array}{c}
k \\
j
\end{array}\right) z^{j} M_{h}^{k-j}\right] d F_{M_{x}}(z)
\end{aligned}
$$

and as $M_{x}^{j}$ and $M_{h}^{k-j}$ are integrable with respect to $F_{Z} \times F_{M_{x}}$, and so it is possible to use the Fubini's 
theorem, to interchange the integral with the expect value:

$$
\begin{aligned}
& =E\left[\sum_{j=0}^{k}\left(\begin{array}{l}
k \\
j
\end{array}\right)\left[\int_{0}^{\infty} z^{j} d F_{M_{x}}(z)\right] M_{h}^{k-j}\right] \\
& =E\left[\sum_{j=0}^{k}\left(\begin{array}{l}
k \\
j
\end{array}\right) E\left[M_{x}^{j}\right] M_{h}^{k-j}\right] \\
& =\sum_{j=0}^{k}\left(\begin{array}{l}
k \\
j
\end{array}\right) E\left[M_{x}^{j}\right] E\left[M_{h}^{k-j}\right] .
\end{aligned}
$$

Lemma 13. For $x>0$, and $k \in \mathbb{Z}^{+}$,

$$
E\left[M_{x}^{k}\right]=\sum_{j=0}^{k-1}\left(\begin{array}{c}
k \\
j
\end{array}\right)\left(\lim _{h \rightarrow 0} \frac{M_{h}^{k-j}}{h}\right) \int_{0}^{x} E\left[M_{s}^{j}\right] d s .
$$

Proof. Let $f_{k}$ be the function defined by $f_{k}(x):=$ $E\left[M_{x}^{k}\right]$ for $x \geq 0$ and $k \in \mathbb{Z}^{+}$, and $f_{0}(x)=1$ for $x \geq 0$. Then,

$$
\begin{aligned}
& f_{k}(x+h)-f_{k}(x) \stackrel{(32)}{=} \\
&\left(\sum_{j=0}^{k}\left(\begin{array}{l}
k \\
j
\end{array}\right) E\left[M_{x}^{j}\right] E\left[M_{h}^{k-j}\right]\right)-E\left[M_{x}^{k}\right], \\
&=\sum_{j=0}^{k-1}\left(\begin{array}{l}
k \\
j
\end{array}\right) E\left[M_{x}^{j}\right] E\left[M_{h}^{k-j}\right] \\
&=\sum_{j=0}^{k-1}\left(\begin{array}{l}
k \\
j
\end{array}\right) f_{j}(x) f_{k-j}(h) .
\end{aligned}
$$

We assume that the limit exists, then,

$$
\begin{aligned}
f_{k}^{\prime}(x) \stackrel{(34)}{=} \lim _{h \rightarrow 0} \frac{\sum_{j=0}^{k-1}\left(\begin{array}{l}
k \\
j
\end{array}\right) f_{j}(x) f_{k-j}(h)}{h}, \\
=\sum_{j=0}^{k-1}\left(\begin{array}{l}
k \\
j
\end{array}\right) f_{j}(x) \lim _{h \rightarrow 0} \frac{f_{k-j}(h)}{h},
\end{aligned}
$$

and given that $f_{k}(0)=E\left[M_{0}^{k}\right]=0$, for all $k \in \mathbb{Z}^{+}$, then

$$
\begin{aligned}
f_{k}(x) & =\int_{0}^{x} f_{k}^{\prime}(s) d s \\
& =\sum_{j=0}^{k-1}\left(\begin{array}{l}
k \\
j
\end{array}\right)\left(\lim _{h \rightarrow 0} \frac{f_{k-j}(h)}{h}\right) \int_{0}^{x} f_{j}(s) d s .
\end{aligned}
$$

\section{Lemma 14.}

$$
\lim _{h \rightarrow 0^{+}} \frac{E\left[M_{h}\right]}{h}=\frac{\lambda_{0}+\lambda_{1}}{\lambda_{0}},
$$

$$
\text { and for } k=2,3, \ldots, \quad \lim _{h \rightarrow 0^{+}} \frac{E\left[M_{h}^{k}\right]}{h}=\frac{k ! \lambda_{1}}{\lambda_{0}^{k}} \text {. }
$$

Proof. Given that $P_{r}\left\{Z_{0}=1\right\}=1$, so let $X_{1}$ represents this first sojourn time in state 1 , and $X_{0}$ represents this first sojourn time in state 0 . So if $h>0$, then

$$
\begin{gathered}
E\left[M_{h}^{k}\right]=E\left[M_{h}^{k} \cdot\left(I_{\left[\left\{X_{1}>h\right\} \cup\left\{X_{1} \leq h\right\}\right]}\right)\right] \\
=E\left[M_{h}^{k} \cdot I_{\left\{X_{1}>h\right\}}\right]+E\left[M_{h}^{k} \cdot I_{\left\{X_{1} \leq h\right\}}\right] \\
=E\left[M_{h}^{k} \cdot I_{\left.\left\{X_{1}>h\right\}\right]}+E\left[M_{h}^{k} \cdot I_{\left[\left\{X_{1} \leq h\right\} \cap\left\{X_{0}+X_{1}>h\right\}\right]}\right]\right. \\
+E\left[M_{h}^{k} \cdot I_{\left[\left\{X_{1} \leq h\right\} \cap\left\{X_{0}+X_{1} \leq h\right\}\right]}\right] \\
=E\left[M_{h}^{k} \cdot I_{\left\{X_{1}>h\right\}}\right]+E\left[M_{h}^{k} \cdot I_{\left\{X_{1} \leq h, X_{0}+X_{1}>h\right\}}\right] \\
+E\left[M_{h}^{k} \cdot I_{\left\{X_{0}+X_{1} \leq h\right\}}\right] .
\end{gathered}
$$

Now, if the first working sojourn time of the system $X_{1}>h$, then, $M_{h}=h$. Thus,

$$
E\left[M_{h}^{k} \cdot I_{\left\{X_{1}>h\right\}}\right]=h^{k} P_{r}\left(X_{1}>h\right)=h^{k} e^{-\lambda_{1} h} .
$$

Also, if $X_{1}+X_{0} \leq h$, then $M_{h}=X_{1}+X_{0}+M_{h-X_{1}}$ and so,

$$
\begin{gathered}
E\left[M_{h}^{k} \cdot I_{\left\{X_{0}+X_{1} \leq h\right\}}\right]= \\
\int_{0}^{h} \int_{0}^{h-x_{1}} \int_{x_{0}+x_{1}}^{\infty} t^{k} \lambda_{0} e^{-\lambda_{0} x_{0}} \lambda_{1} e^{-\lambda_{1} x_{1}} d F_{M_{h}}(t) d x_{0} d x_{1} \\
=\int_{0}^{h} \int_{0}^{h-x_{1}} \int_{0}^{\infty}\left(x_{0}+x_{1}+y\right)^{k} \lambda_{0} e^{-\lambda_{0} x_{0}} \\
\cdot \lambda_{1} e^{-\lambda_{1} x_{1}} d F_{M_{h-x_{1}}}(y) d x_{0} d x_{1} \\
\leq \int_{0}^{h} \int_{0}^{h-x_{1}} \int_{0}^{\infty}(h+t)^{k} \lambda_{0} e^{-\lambda_{0} x_{0}} \\
\cdot \lambda_{1} e^{-\lambda_{1} x_{1}} d F_{M_{h}}(t) d x_{0} d x_{1} \\
=\int_{0}^{h} \int_{0}^{h-x_{1}} E\left[\left(h+M_{h}\right)^{k}\right] \lambda_{0} e^{-\lambda_{0} x_{0}} \lambda_{1} e^{-\lambda_{1} x_{1}} d x_{0} d x_{1} \\
=E\left[\left(h+M_{h}\right)^{k}\right] \cdot P_{r}\left(X_{1}+X_{0} \leq h\right)=o(h), \quad(37)
\end{gathered}
$$

since $P_{r}\left(X_{1}+X_{0} \leq h\right)=o(h)$ and by the dominated convergence theorem,

$$
\lim _{h \rightarrow 0^{+}} E\left[\left(h+M_{h}\right)^{k}\right]=0 .
$$


Besides, if $X_{1} \leq h$ and $X_{0}+X_{1}>h$, then $M_{h}=$ $X_{1}+X_{0}+M_{h-X_{1}}$ and so,

$$
\begin{aligned}
& E\left[M_{h}^{k} \cdot I_{\left\{X_{1} \leq h, X_{1}+X_{0}>h\right\}}\right]= \\
& \int_{0}^{h} \int_{h-x_{1}}^{\infty} \int_{0}^{\infty} t^{k} \lambda_{0} e^{-\lambda_{0} x_{0}} \lambda_{1} e^{-\lambda_{1} x_{1}} d F_{M_{h}}(t) d x_{0} d x_{1} \\
& =\int_{0}^{h} \int_{h-x_{1}}^{\infty} E\left[M_{h}^{k} \mid X_{1}=x_{1}, X_{0}=x_{0}\right] \\
& \text { - } \lambda_{0} e^{-\lambda_{0} x_{0}} \lambda_{1} e^{-\lambda_{1} x_{1}} d x_{0} d x_{1} \\
& =\int_{0}^{h} \int_{h-x_{1}}^{\infty} E\left[\left(x_{0}+x_{1}+M_{h-x_{1}}\right)^{k}\right] \\
& \text { - } \lambda_{0} e^{-\lambda_{0} x_{0}} \lambda_{1} e^{-\lambda_{1} x_{1}} d x_{0} d x_{1} \\
& =\int_{0}^{h} \int_{h-x_{1}}^{\infty} \sum_{j=0}^{k}\left(\begin{array}{l}
k \\
j
\end{array}\right) E\left[\left(x_{1}+M_{h-x_{1}}\right)^{j}\right] \\
& \cdot x_{0}^{k-j} \lambda_{0} e^{-\lambda_{0} x_{0}} \lambda_{1} e^{-\lambda_{1} x_{1}} d x_{0} d x_{1} \\
& =\int_{0}^{h} \sum_{j=0}^{k}\left(\begin{array}{l}
k \\
j
\end{array}\right) E\left[\left(x_{1}+M_{h-x_{1}}\right)^{j}\right] \\
& \cdot\left[\int_{h-x_{1}}^{\infty} x_{0}^{k-j} \lambda_{0} e^{-\lambda_{0} x_{0}} d x_{0}\right] \lambda_{1} e^{-\lambda_{1} x_{1}} d x_{1} \\
& =\int_{0}^{h} \sum_{j=0}^{k}\left(\begin{array}{l}
k \\
j
\end{array}\right) E\left[\left(x_{1}+M_{h-x_{1}}\right)^{j}\right] \\
& \cdot\left[\int_{0}^{\infty}\left(y+h-x_{1}\right)^{k-j} \lambda_{0} e^{-\lambda_{0}\left(y+h-x_{1}\right)} d y\right] \\
& \cdot \lambda_{1} e^{-\lambda_{1} x_{1}} d x_{1} \\
& =\int_{0}^{h} \sum_{j=0}^{k}\left(\begin{array}{l}
k \\
j
\end{array}\right) E\left[\left(x_{1}+M_{h-x_{1}}\right)^{j}\right] \\
& \cdot\left[\int_{0}^{\infty}\left(y+h-x_{1}\right)^{k-j} \lambda_{0} e^{-\lambda_{0} y} d y\right] \\
& \cdot e^{-\lambda_{0}\left(h-x_{1}\right)} \lambda_{1} e^{-\lambda_{1} x_{1}} d x_{1} \\
& =\int_{0}^{h} \sum_{j=0}^{k}\left(\begin{array}{l}
k \\
j
\end{array}\right) E\left[\left(x_{1}+M_{h-x_{1}}\right)^{j}\right] \\
& E\left[\left(X_{0}+h-x_{1}\right)^{k-j}\right] \\
& \cdot e^{-\lambda_{0}\left(h-x_{1}\right)} \lambda_{1} e^{-\lambda_{1} x_{1}} d x_{1},
\end{aligned}
$$

and substituting $z=h-x_{1}$, $=$

$$
\begin{gathered}
\int_{0}^{h} \sum_{j=0}^{k}\left(\begin{array}{l}
k \\
j
\end{array}\right) E\left[\left(h-z+M_{z}\right)^{j}\right] e^{-\lambda_{0} z} \\
\cdot E\left[\left(X_{0}+z\right)^{k-j}\right] \lambda_{1} e^{-\lambda_{1}(h-z)} d z \\
=\int_{0}^{h} \sum_{j=0}^{k}\left(\begin{array}{l}
k \\
j
\end{array}\right) \sum_{i=0}^{j}\left(\begin{array}{l}
j \\
i
\end{array}\right) h^{i} E\left[\left(M_{z}-z\right)^{j-i}\right] e^{-\lambda_{0} z} \\
\cdot E\left[\left(X_{0}+z\right)^{k-j}\right] \cdot \lambda_{1} e^{-\lambda_{1}(h-z)} d z=
\end{gathered}
$$

$$
\begin{aligned}
& \sum_{j=0}^{k} \sum_{i=0}^{j}\left(\begin{array}{l}
k \\
j
\end{array}\right)\left(\begin{array}{l}
j \\
i
\end{array}\right) h^{i} \lambda_{1} e^{-\lambda_{1} h} \int_{0}^{h} E\left[\left(M_{z}-z\right)^{j-i}\right] \\
& \cdot e^{-\left(\lambda_{0}-\lambda_{1}\right) z} \cdot E\left[\left(X_{0}+z\right)^{k-j}\right] d z .
\end{aligned}
$$

Now, if $i<j$ then,

$$
\begin{gathered}
\left|\int_{0}^{h} E\left[\left(M_{z}-z\right)^{j-i}\right] e^{-\left(\lambda_{0}-\lambda_{1}\right) z} E\left[\left(X_{0}+z\right)^{k-j}\right] d z\right| \\
\leq \int_{0}^{h} E\left[M_{h}^{j-i}\right] e^{-\left(\lambda_{0}-\lambda_{1}\right) z} E\left[\left(X_{0}+h\right)^{k-j}\right] d z \\
\leq \int_{0}^{h} E\left[M_{h}^{j-i}\right] e^{-\left(\lambda_{0}-\lambda_{1}\right) z} E\left[(2 h)^{k-j}\right] d z \\
=E\left[M_{h}^{j-i}\right]\left[\frac{\lambda_{1}}{\lambda_{0}}\left(1-e^{-\lambda_{0} h}\right)\right](2 h)^{k-j} \\
\leq m h^{j-i}\left[\frac{\lambda_{1}}{\lambda_{0}}\left(1-e^{-\lambda_{0} h}\right)\right](2 h)^{k-j},
\end{gathered}
$$

for some $m \in \mathbb{Z}^{+}$, and so,

$$
\begin{aligned}
& \lim _{h \rightarrow 0^{+}} \frac{1}{h} \int_{0}^{h} E\left[\left(M_{z}-z\right)^{j-i}\right] \\
& \cdot e^{-\left(\lambda_{0}-\lambda_{1}\right) z} E\left[\left(X_{0}+z\right)^{k-j}\right] d z=0,
\end{aligned}
$$

but if $i=j$ then,

$$
\begin{aligned}
& \int_{0}^{h} e^{-\left(\lambda_{0}-\lambda_{1}\right) z} E\left[\left(X_{0}+z\right)^{k-j}\right] d z \\
= & \int_{0}^{h} e^{-\left(\lambda_{0}-\lambda_{1}\right) z} \int_{0}^{\infty}\left(x_{0}+z\right)^{k-j} \lambda_{0} e^{-\lambda_{0} x_{0}} d x_{0} d z,
\end{aligned}
$$

and set $y=x_{0}+z$,

$$
=\int_{0}^{h} e^{\lambda_{1} z} \int_{z}^{\infty} \lambda_{0} e^{-\lambda_{0} y} y^{k-j} d y d z
$$

But,

$$
\begin{aligned}
& \int_{z}^{\infty} \lambda_{0} e^{-\lambda_{0} y} y^{k-j} d y= \\
& \int_{0}^{\infty} \lambda_{0} e^{-\lambda_{0} y} y^{k-j} d y-\int_{0}^{z} \lambda_{0} e^{-\lambda_{0} y} y^{k-j} d y= \\
& E\left[X_{0}^{k-j}\right]-\sum_{n=0}^{\infty} \frac{(-1)^{n} \lambda_{0}^{n+1} z^{k-j+n+1}}{n !(k-j+n+1)} .
\end{aligned}
$$

taking $a_{n}=\frac{(-1)^{n} \lambda_{0}^{n+1}}{n !(k-j+n+1)}$, and by using (40),

$$
\begin{aligned}
& \int_{0}^{h} e^{-\left(\lambda_{0}-\lambda_{1}\right) z} E\left[\left(X_{0}+z\right)^{k-j}\right] d z= \\
& \int_{0}^{h} e^{\lambda_{1} z}\left[E\left[X_{0}^{k-j}\right]-\sum_{n=0}^{\infty} a_{n} z^{k-j+n+1}\right] d z= \\
& E\left[X_{0}^{k-j}\right] \frac{e^{\lambda_{1} h}-1}{\lambda_{1}}-o(h),
\end{aligned}
$$


thus,

$$
\begin{array}{r}
\lim _{h \rightarrow 0^{+}} \frac{1}{h} \int_{0}^{h} e^{-\left(\lambda_{0}-\lambda_{1}\right) z} E\left[\left(X_{0}+z\right)^{k-j}\right] d z= \\
E\left[X_{0}^{k-j}\right]=\frac{(k-j) !}{\lambda_{0}^{k-j}} .
\end{array}
$$

Then by (38), (39) and (41), it is obtained,

$$
\lim _{h \rightarrow 0^{+}} \frac{1}{h} E\left[M_{h}^{k} \cdot I_{\left\{X_{1} \leq h, X_{1}+X_{0}>h\right\}}\right]=\frac{k ! \lambda_{1}}{\lambda_{0}^{k}} .
$$

Then, if $k=1$, by (36),

$$
\lim _{h \rightarrow 0^{+}} \frac{1}{h} E\left[M_{h} \cdot I_{\left\{X_{1}>h\right\}}\right]=\lim _{h \rightarrow 0^{+}} \frac{1}{h} h e^{-\lambda_{1} h}=1,
$$

by (42),

$$
\lim _{h \rightarrow 0^{+}} \frac{1}{h} E\left[M_{h} \cdot I_{\left\{X_{1} \leq h, X_{1}+X_{0}>h\right\}}\right]=\frac{\lambda_{1}}{\lambda_{0}},
$$

and combining these two latest results with (37), it is obtained that,

$$
\lim _{h \rightarrow 0^{+}} \frac{1}{h} E\left[M_{h}\right]=1+\frac{\lambda_{1}}{\lambda_{0}}=\frac{\lambda_{0}+\lambda_{1}}{\lambda_{0}} .
$$

Now, if $k \geq 2$, by using (36), (37) and (42), it results that

$$
\lim _{h \rightarrow 0^{+}} \frac{1}{h} E\left[M_{h}^{k}\right]=\frac{k ! \lambda_{1}}{\lambda_{0}^{k}} .
$$

Theorem 15. For all $x>0$, it is true the next recurrent formula:

$$
E\left[M_{x}\right]=\left(\frac{\lambda_{0}+\lambda_{1}}{\lambda_{0}}\right) x
$$

and for $k=2,3, \ldots$,

$$
\begin{array}{r}
E\left[M_{x}^{k}\right]=\sum_{j=0}^{k-2}\left(\begin{array}{l}
k \\
j
\end{array}\right)\left[\frac{(k-j) ! \lambda_{1}}{\lambda_{0}^{k-j}}\right] \int_{0}^{x} E\left[M_{s}^{j}\right] d s \\
+k\left[\frac{\lambda_{0}+\lambda_{1}}{\lambda_{0}}\right] \int_{0}^{x} E\left[M_{s}^{k-1}\right] d s .
\end{array}
$$

Proof. The proof follows from the Lemma 13 and Lemma 14.

Example 16. Using Theorem 15, we have

$$
E\left[M_{x}\right]=\frac{\lambda_{0}+\lambda_{1}}{\lambda_{0}} x, \quad \text { and }
$$

$$
\begin{aligned}
& E\left[M_{x}^{2}\right]= \\
& \begin{array}{r}
\frac{2 \lambda_{1}}{\lambda_{0}^{2}} \int_{0}^{x} E\left[M_{s}^{0}\right] d s+2 \frac{\lambda_{0}+\lambda_{1}}{\lambda_{0}} \int_{0}^{x} E\left[M_{s}\right] s d s= \\
\frac{2 \lambda_{1}}{\lambda_{0}^{2}} x+2 \frac{\lambda_{0}+\lambda_{1}}{\lambda_{0}} \int_{0}^{x}\left[\frac{\lambda_{0}+\lambda_{1}}{\lambda_{0}}\right] s d s= \\
\frac{2 \lambda_{1}}{\lambda_{0}^{2}} x+\left(\frac{\lambda_{0}+\lambda_{1}}{\lambda_{0}}\right)^{2} x^{2}
\end{array}
\end{aligned}
$$

and so,

$$
\operatorname{Var}\left[M_{x}\right]=E\left[M_{x}^{2}\right]-E\left[M_{x}\right]^{2}=\frac{2 \lambda_{1}}{\lambda_{0}^{2}} x .
$$

These are the same results that in Theorem 8 .

Corollary 17. For all $k \in \mathbb{Z}^{+}$, and $x>0, E\left[M_{x}^{k}\right]$ is a polynomial of degree $k$, more precisely

$$
E\left[M_{x}^{k}\right]=\sum_{i=0}^{k} c_{k i} x^{i}
$$

for some $c_{k 0}, c_{k 1}, \ldots, c_{k k} \in \mathbb{R}$, where

$$
c_{k k}=\left(\frac{\lambda_{0}+\lambda_{1}}{\lambda_{0}}\right)^{k} \text {. }
$$

Proof. By induction: In Example 16, it was seen the cases for $k=1$ and $k=2$. Let $n \in\{2,3, \ldots\}$ and suppose that for $j=0,1, \ldots, n, E\left[M_{x}^{j}\right]=\sum_{i=0}^{j} c_{j i} x^{i}$, for some $c_{j i} \in \mathbb{R}$, where $c_{j j}=\left(\frac{\lambda_{0}+\lambda_{1}}{\lambda_{0}}\right)^{j}$. Then, by Theorem 15,

$$
\begin{aligned}
& E\left[M_{x}^{n+1}\right]=\sum_{j=0}^{n-1}\left(\begin{array}{c}
n+1 \\
j
\end{array}\right)\left[\frac{(n+1-j) ! \lambda_{1}}{\lambda_{0}^{n+1-j}}\right] \\
& \times \int_{0}^{x} E\left[M_{s}^{j}\right] d s+(n+1)\left(\frac{\lambda_{0}+\lambda_{1}}{\lambda_{0}}\right) \int_{0}^{x} E\left[M_{s}^{n}\right] d s \\
& =\sum_{j=0}^{n-1}\left(\begin{array}{c}
n+1 \\
j
\end{array}\right)\left[\frac{(n+1-j) ! \lambda_{1}}{\lambda_{0}^{n+1-j}}\right] \int_{0}^{x} \sum_{i=0}^{j} c_{j i} s^{i} d s \\
& +(n+1)\left(\frac{\lambda_{0}+\lambda_{1}}{\lambda_{0}}\right) \int_{0}^{x} \sum_{i=0}^{n} c_{n i} s^{i} d s \\
& =\sum_{j=0}^{n-1}\left(\begin{array}{c}
n+1 \\
j
\end{array}\right)\left[\frac{(n+1-j) ! \lambda_{1}}{\lambda_{0}^{n+1-j}}\right] \sum_{i=0}^{j} \frac{c_{j i}}{i+1} x^{i+1} \\
& +(n+1)\left(\frac{\lambda_{0}+\lambda_{1}}{\lambda_{0}}\right) \sum_{i=0}^{n} \frac{c_{n i}}{i+1} x^{i+1} \\
& =q(x)+(n+1)\left(\frac{\lambda_{0}+\lambda_{1}}{\lambda_{0}}\right) \frac{c_{n i}}{n+1} x^{n+1}, \\
& =q(x)+\left(\frac{\lambda_{0}+\lambda_{1}}{\lambda_{0}}\right)^{n+1} c_{n i} x^{n+1},
\end{aligned}
$$

where $q(x)$ is a polynomial of degree $\mathrm{n}$. 
Corollary 18. For all $k \in \mathbb{Z}^{+}$,

$$
\lim _{x \rightarrow \infty} \frac{E\left[M_{x}^{k}\right]}{x^{k}}=\left[\frac{\lambda_{0}+\lambda_{1}}{\lambda_{0}}\right]^{k} .
$$

Proof. If $k \in \mathbb{Z}^{+}$, then by Corollary 17 ,

$$
\lim _{x \rightarrow \infty} \frac{E\left[M_{x}^{k}\right]}{x^{k}}=\lim _{x \rightarrow \infty} \frac{p(x)+\left(\frac{\lambda_{0}+\lambda_{1}}{\lambda_{0}}\right)^{k} x^{k}}{x^{k}},
$$

where $p(x)$ is a polynomial of degree $k-1$, (see [12]),

$$
=\lim _{x \rightarrow \infty}\left[\frac{p(x)}{x^{k}}+\frac{\left(\frac{\lambda_{0}+\lambda_{1}}{\lambda_{0}}\right)^{k} x^{k}}{x^{k}}\right]=\left(\frac{\lambda_{0}+\lambda_{1}}{\lambda_{0}}\right)^{k},
$$

which coincides with the asymptotic results in Corollary 9 .

\subsection{The Moment Generating Function of $M_{x}$}

We now give an another approach to derive the moments for the mission time $E\left[M_{x}^{k}\right]$, for $x \geq 0$, using the fact that the process has the independent increments, the distribution of $M_{x}$ is fully represented. Let us take $U_{1}$ the random time that the system is working for the first time, and $D_{1}$ be the random time that the system is down immediately after a time $U_{1}$. In general, $U_{i}$ is the random time at which the system is working, and $D_{i}$ is the random time at which the system is down immediately after a time $U_{i}$, for $i=1,2, \ldots$. Note that both $U_{i}$ 's and $D_{i}$ are independent and identically distributed exponential random variables with parameters $\lambda_{1}$ and $\lambda_{0}$ respectively. We now define

$$
\begin{aligned}
X_{1} & =U_{1}, \\
X_{i} & =D_{i-1}+U_{i}, \quad i=2,3, \ldots,
\end{aligned}
$$

Let $T_{n}=X_{1}+X_{2}+\cdots+X_{n}$, for $n=1,2 \ldots$, then $\left\{T_{n} ; n=1,2, \ldots\right\}$ is a delayed renewal process and its counting process $\left\{N_{T}(t) ; t \geq 0\right\}$, represents the number of times the systems failed during the time interval $[0, t]$. Let $S_{n}=U_{1}+U_{2}+\cdots+U_{n}$, for $n=$ $1,2 \ldots$, then $\left\{S_{n} ; n=1,2, \ldots\right\}$ be an renewal process, which represents the times at which after each failure and the subsequent replacement by a new component. The counting process for $\left\{S_{n} ; n=1,2, \ldots\right\}$ is denoted by $\{N(t) ; t \geq 0\}$. Thus, for $x \geq 0$ fixed time and $M_{x}$ is the random mission time, and if we subtract $M_{x}$ from $D_{1}+\cdots+D_{N_{T}\left(M_{x}\right)}$ (the times at which the system is not working until $M_{x}$ ), we get

$$
N_{T}\left(M_{x}\right)=N(x) \text {, almost everywhere }(a e),
$$

this is due to the fact that

$$
\begin{array}{r}
U_{1}+\left(D_{1}+U_{2}\right)+\cdots+\left(D_{N_{T}\left(M_{x}\right)-1}+U_{N_{T}\left(M_{x}\right)}\right)+ \\
{\left[D_{N_{T}\left(M_{x}\right)}+\left(x-\sum_{i=1}^{N_{T}\left(M_{x}\right)} U_{i}\right)\right] \stackrel{a e}{=} M_{x},}
\end{array}
$$

so that,

$$
x+\sum_{i=1}^{N(x)} D_{i} \stackrel{a e}{=} M_{x} .
$$

Now, by using the Wald's equation, we obtain:

$$
\begin{aligned}
E\left[M_{x}\right] & =x+E[N(x)] \cdot E\left[D_{1}\right] \\
& =x+\left[\lambda_{1} x\right] \cdot\left[\frac{1}{\lambda_{0}}\right] \\
& =\left(1+\frac{\lambda_{1}}{\lambda_{0}}\right) x .
\end{aligned}
$$

If $\phi_{X}(t)$ represents the moment generating function of a random variable $X$, i.e,

$$
\phi_{X}(t)=E\left[e^{t X}\right],
$$

then,

$$
\begin{aligned}
\phi_{M_{x}}(t) & =E\left[e^{t M_{x}}\right]=E\left[e^{t\left(x+\sum_{i=1}^{N(x)} D_{i}\right)}\right] \\
& =e^{t x} E\left[\prod_{i=1}^{N(x)} e^{t D_{i}}\right] \\
& =e^{t x} \sum_{n=0}^{\infty} E\left[\prod_{i=1}^{N(x)} e^{t D_{i}} \mid N(x)=n\right] \cdot P_{r}(N(x)=n) \\
& =e^{t x} \sum_{n=0}^{\infty} E\left[\prod_{i=1}^{n} e^{t D_{i}}\right] \cdot\left(e^{-\lambda_{1} x} \frac{\left(\lambda_{1} x\right)^{n}}{n !}\right) \\
& =e^{t x-\lambda_{1} x} \sum_{n=0}^{\infty} \frac{\left(E\left[e^{t D_{1}}\right] \lambda_{1} x\right)^{n}}{n !} \\
& =e^{t x-\lambda_{1} x} e^{\phi_{D_{1}}(t) \lambda_{1} x} \\
& =\exp \left(x\left[t-\lambda_{1}+\left[1-\frac{t}{\lambda_{0}}\right]^{-1} \lambda_{1}\right]\right) .
\end{aligned}
$$

Then,

$$
\begin{aligned}
& \phi_{M_{x}}^{\prime}(t)=x \cdot \phi_{M_{x}}(t) \cdot \frac{\lambda_{0} \lambda_{1}+\left(t-\lambda_{0}\right)^{2}}{\left(t-\lambda_{0}\right)^{2}}, \text { and so, } \\
& \phi_{M_{x}}^{\prime}(0)=E\left[M_{x}\right]=\frac{\lambda_{0}+\lambda_{1}}{\lambda_{0}} x .
\end{aligned}
$$


And

$$
\phi_{M_{x}}^{\prime \prime}(t)=x \cdot \phi_{M_{x}}(t) \cdot \frac{x\left(\left(t-\lambda_{0}\right)^{2}+\lambda_{0} \lambda_{1}\right)^{2}-2 \lambda_{0} \lambda_{1}\left(t-\lambda_{0}\right)}{\left(t-\lambda_{0}\right)^{4}},
$$

thus,

$$
\phi_{M_{x}}^{\prime \prime}(0)=E\left[M_{x}^{2}\right]=\frac{2 \lambda_{1} x}{\lambda_{0}^{2}}+\left(\frac{\lambda_{0}+\lambda_{1}}{\lambda_{0}}\right)^{2} x^{2},
$$

which coincides with the results in Theorem 8 .

Remark 19. It is known that as $x \rightarrow \infty$,

$$
\frac{M_{x}}{x} \rightarrow \frac{\lambda_{0}+\lambda_{1}}{\lambda_{0}}
$$

almost surely, which is true because

$$
\lim _{x \rightarrow \infty} \frac{x}{M_{x}}=\lim _{t \rightarrow \infty} \frac{1}{t} \int_{0}^{t} I_{\{Z(s)=1\}} d s
$$

where both limits represent the long-run fraction of time the environment process $Z$ is in state 1 . Clearly the limit on the right-hand-side of this equality converges almost surely to $\lambda_{0} /\left(\lambda_{0}+\lambda_{1}\right)$ since $Z$ is both irreducible and positive.

Remark 20. In [8], the Laplace transform (with respect to $x$ ) of $E\left[M_{x}^{k}\right]$ is computed, and asymptotic (as $x \rightarrow \infty)$ are derived for both the first and second moments of $M_{x}$. In fact, if the state space $S$ of $\{Z(t) ; t \geq 0\}$ is an arbitrary finite state space, with $C_{t}$ satisfying

$$
C_{t}=\int_{0}^{t} \sum_{i \in S} V_{i} I_{\left\{Z_{h}=i\right\}} d h
$$

and $M_{x}$ defined in the same way and it is assumed that $V_{i}>0$ for all $i$, however, in this paper the case where $V_{1}>0, V_{0}=0$ is considered. By think studying a more general CTMC $\{Z(t) ; t \geq 0\}$ whose state space $S$ is finite, and can be decomposed as $S=S_{+} \cup S_{0}$, where

$$
S_{+}=\left\{i \in S: V_{i}>0\right\}, S_{0}=\left\{i \in S: V_{i}=0\right\} .
$$

This is more general than the model considered in [8], and it should be possible to study the moments of $M_{x}$ by using the results of this Section. These same ideas could possibly be used to study the moments of $M_{x}$ when $S_{+}$contains two or more elements as well. Furthermore, considering cases where $S_{0}$ contains many elements would correspond to downtimes that are phase-type distributed, and this is interesting from a reliability perspective since it allows us to model downtimes that are not well-described by exponential random variables.

\section{Numerical Illustration}

In this section, we analyze, through illustrations, what happens to the expected value and variance of the mission time $M_{x}$, when we choose different lengths of the mission $x$ and different values for $\lambda_{0}$ and $\lambda_{1}$.

Since our interest is to analyze the overall behavior of these measures, we interpret the random variable $M_{x}$, the expected value $E\left[M_{x}\right]$ and the variance $\operatorname{Var}\left[M_{x}\right]$ as functions of the length of the mission $x$ with the following functions:

$y(x)=\mu_{x}, y_{1}(x)=\mu_{x} \pm \sigma_{x}, y_{2}(x)=\mu_{x} \pm 2 \sigma_{x}$ and $y_{3}(x)=\mu_{x} \pm 3 \sigma_{x}$, where $\mu_{x}=m_{1}(x)=E\left[M_{x}\right]$ and $\sigma_{x}=\sqrt{\operatorname{Var}\left[M_{x}\right]}$.

The purpose of defining the above functions is to observe what happens to the behavior of the random variable $M_{x}$, for each value of $x \geq 0$. In fact, we observe how the intervals $\left(\mu_{x}-\sigma_{x}, \mu_{x}+\sigma_{x}\right)$, $\left(\mu_{x}-2 \sigma_{x}, \mu_{x}+2 \sigma_{x}\right)$ and $\left(\mu_{x}-3 \sigma_{x}, \mu_{x}+3 \sigma_{x}\right)$ behave, because it is known that at these intervals most of the information about $M_{x}$ is concentrated. In each of the Figures 1 to 9, we show the graphs of $y(x)$, $y_{1}(x), y_{2}(x)$ and $y_{3}(x)$, but in each graph, a different analysis is performed according to the variation of the parameters $\lambda_{0}$ and $\lambda_{1}$.

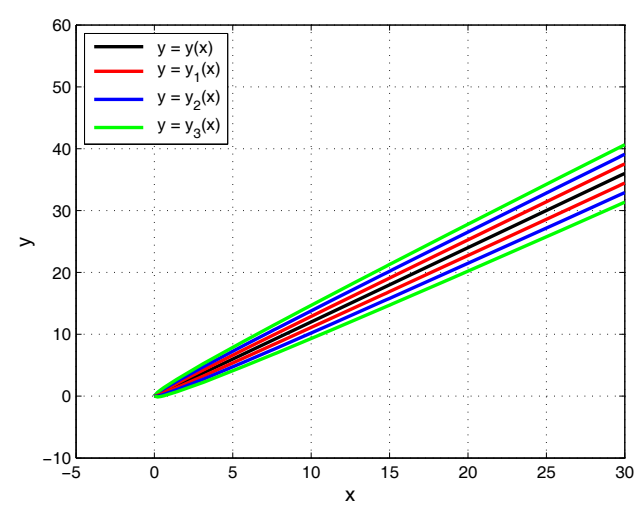

Figure 1. $\lambda_{0}=5.0, \lambda_{1}=1.0$. 


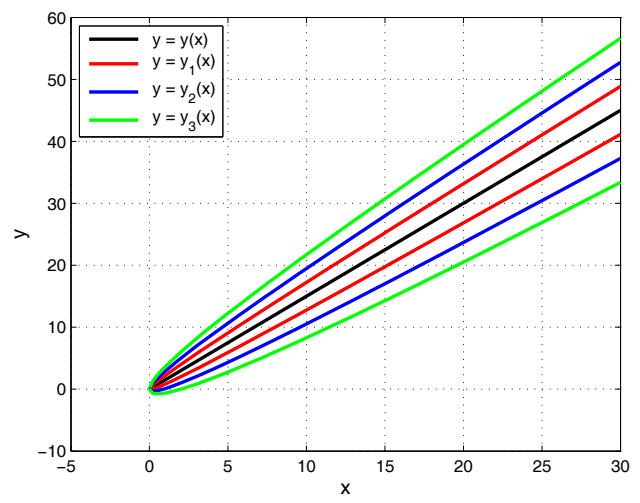

Figure 2. $\lambda_{0}=2.0, \lambda_{1}=1.0$.

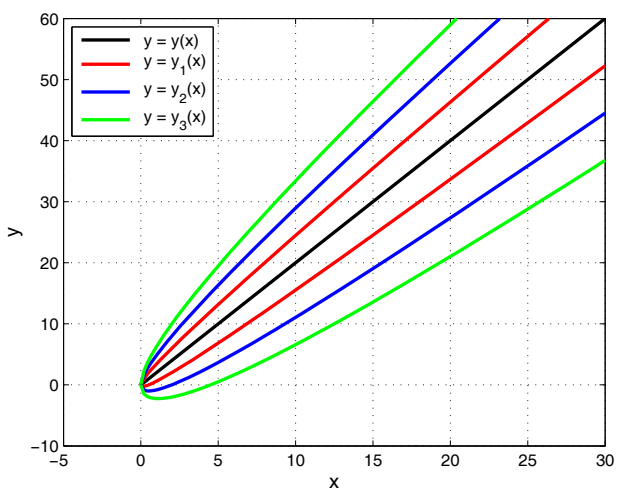

Figure 3. $\lambda_{0}=1.0, \lambda_{1}=1.0$.

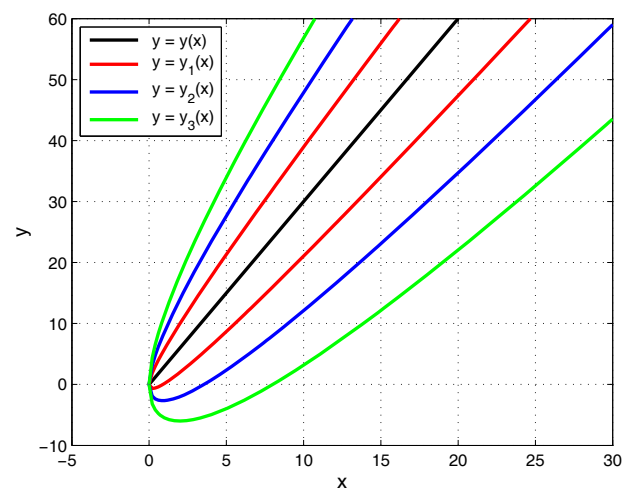

Figure 4. $\lambda_{0}=0.5, \lambda_{1}=1.0$.

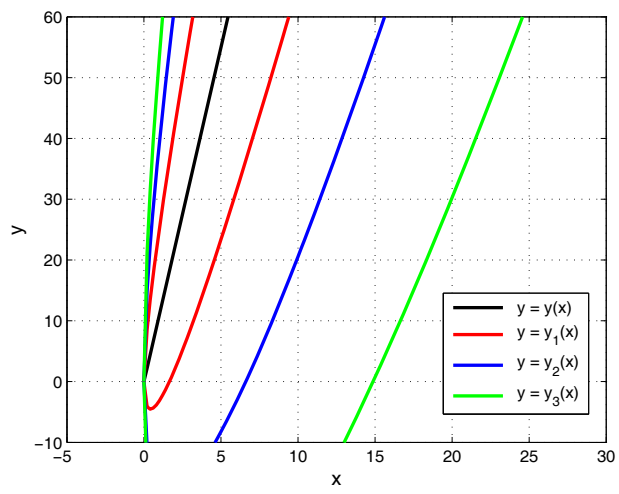

Figure 5. $\lambda_{0}=0.1, \lambda_{1}=1.0$.
In Figures 1 to 5, we are illustrating the situation by means of five graphs, in which $\lambda_{1}=1.0$ is fixed and in each graph $\lambda_{0}$ value is varied from high to low. It is noted that for each fixed value of $x$, if the $\lambda_{0}$ parameter value decreases, then both $m_{1}(x)$ values and the standard deviations of $M_{x}$, increase. In addition it is noted that in each of graphs, if $x$ is increasing then both $m_{1}(x)$ values and the standard deviations of $M_{x}$ also increase.

In Figures 6, 7, 3, 8 and 9, there are five graphs in which $\lambda_{0}=1.0$ is fixed and in each graph we have varied $\lambda_{1}$ value from low to high. It is noted that for each fixed value of $x$, if $\lambda_{1}$ value increases, then both $\mu_{x}$ and $\sigma_{x}$, increase. In addition it is noted that in each of graphs, if $x$ is increasing then both $\mu_{x}$ and $\sigma_{x}$ also increase.

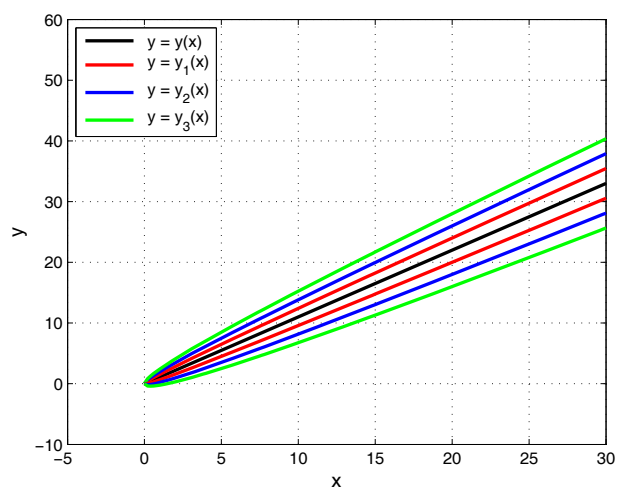

Figure 6. $\lambda_{0}=1.0, \lambda_{1}=0.1$.

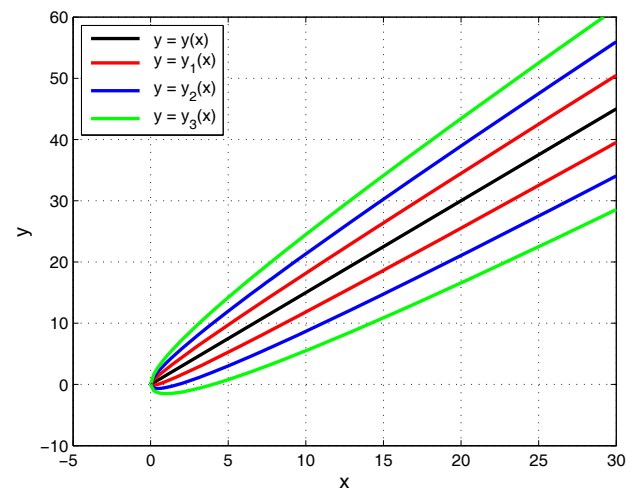

Figure 7. $\lambda_{0}=1.0, \lambda_{1}=0.5$.

Cases with large standard deviations happen if either $\lambda_{0}$ values are small or $\lambda_{1}$ values are large. In any case, the most critical situations are observed for small values of $x$, since the coefficients of variation $\left(C V(x)=\sigma_{x} / \mu_{x}\right)$ are very large. In Figures 4 and 5, we observe that the above affirmation is true. For values of $x$ close to 0 , the standard deviation is very 
large in proportion to $\mu_{x}$. In Figures 8 and 9, we realize that for large values of $x$, the standard deviations are very small in proportion to $\mu_{x}$, that is, the coefficients of variation are very small.

From these analyses we conclude that the prediction obtained by estimation of $E\left[M_{x}\right]$ is quite useful, especially because the length of the mission $x$ required in real situations, is not small. It is also noted that the results obtained are better for large values of $\lambda_{0}$ and small values of $\lambda_{1}$.

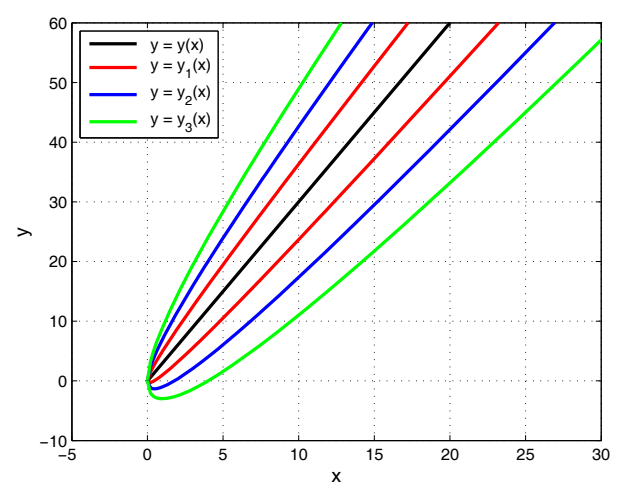

Figure 8. $\lambda_{0}=1.0, \lambda_{1}=2.0$.

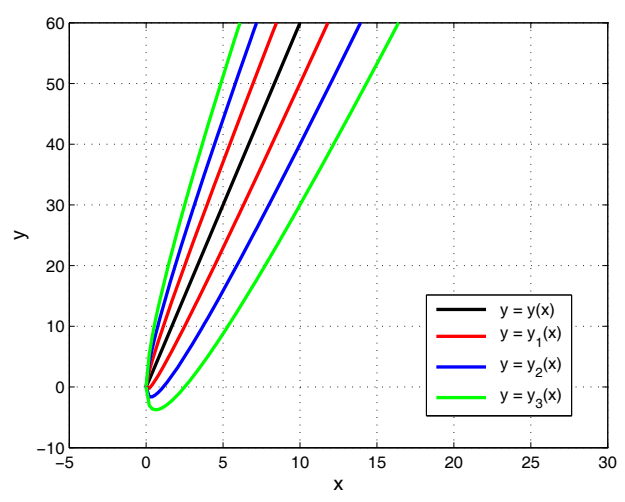

Figure 9. $\lambda_{0}=1.0, \lambda_{1}=5.0$.

\section{Conclusion}

With the increasing complexity and automation related to systems encountered in the modern industries, the random mission time analysis is being recognized as the suitable reliability method for studying system availability. The novelty of this article is to obtain the distribution of the random mission time and its transient and asymptotic moments by two different approaches. In the first approach, the problem was modeled by using the theory of the link travel time and the second approach, we have proposed an alternative direct method to calculate moments of random mission time drifting from the classical theory of probability. An example is presented to evaluate the expected value and variance of the mission time for the system.

\section{References}

[1] V. Arunachalam, A. Calvache, and A. Tansu, "Some Useful Approximations for the Availability Function", International Journal of Reliability, Quality and Safety Engineering, vol. 22, pp. 155008 1-155008 15, 2015.

[2] V. Arunachalam, S. Dharmaraja, "Fluid Queue Driven by Finite State Markov Processes", Ciencia en Desarrollo, vol. 5, no. 2, pp. 7986, 2014.

[3] D.S. Berry, and D.M. Belmont, "Distribution of Vehicle Speeds and Travel Times", Proceedings of the Second Berkeley Symposium on Mathematical Statistics and Probability, pp. 589-602, 1951.

[4] L. Blanco, V. Arunachalam, and S. Dharmaraja, "Introduction to Probability and Stochastic Processes with Applications", Wiley, New Jersey, 2012.

[5] M. Chen, and S. Chien, "Dynamic freeway travel time prediction using probe vehicle data: Link-based vs. Path-based", Transportation Research Record, vol. 1768, pp. 157-161, 2001.

[6] M. D'Angelo, H.M. Haitham, and M.C. Wang, "Travel-time prediction for freeway corridors", Transportation Research Board, vol. 1676, pp. 184-191, 1999.

[7] E. De Souza e Silva, and H. R. Gail, "Calculating Cumulative Operational Time Distributions of Repairable Computer Systems", IEEE, Transactions on Computers vol. 35, pp. 322332, 1986.

[8] L. Donatiello, and B. Iyer, "Closed-Form solution for system availability distribution", IEEE, Transactions on reliability, vol. 36, pp. 45-47, 1987.

[9] A. Goyal, and A.N. Tanwani, "A measure of guaranteed availability and its numerical evaluation", IEEE Transactions on Computers vol. 37, pp. 25-32, 1988.

[10] J. Kharoufeh, and N. Gautam, "A fluid queueing model for link travel time moments", Naval 
Research Logistics Quarterly, vol. 51, pp. 242257, 2004.

[11] J. Kharoufeh, and N. Gautam, "Deriving Link Travel-Time Distributions via Stochastic Speed Processes", Transportation Science, vol. 38, pp. 97-106, 2004.

[12] L. A. Molano, "Interpolación polinomial, algunas técnicas y su programación", Ciencia en Desarrollo, vol. 4, no. 1, pp. 43-69, 2012.

[13] D. Roden, "Forecasting Travel Time", Transportation Research Record, vol. 1518, pp. 7-12, 1996.
[14] G. Rubino, and B. Sericola, "Interval availability analysis using operational periods", Perfomance Evaluation, vol. 14, pp. 257-272, 1992.

[15] B. Sericola, "Closed-Form solution for the distribution of the total time spent in a subset of states of a homogeneous Markov Process during a finite observation period", Journal of Applied Probability, vol. 27, pp. 713-719, 1990.

[16] L. Vanajakshi, S.C. Subramanian, and R. Sivanandan, "Travel time prediction under heterogeneous trafic conditions using global positioning system data from buses", Intelligent Transport Systems, vol. 3, pp. 1-9, 2009. 